\section{Brain, Behavior and Evolution}

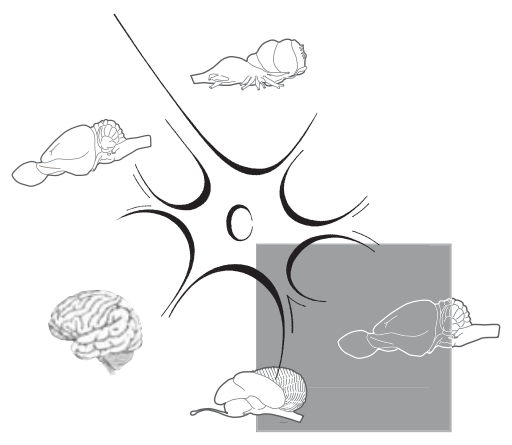

The importance of studying natural populations to understand the basic mechanisms that underlie adult neurogenesis and neuronal replacement and their magnitude has already been emphasized [e.g. Boonstra et al., 2001; Nottebohm, 2002; Amrein et al., 2008; Barnea, 2009]. However, since laboratory studies are both necessary and unavoidable, others also emphasized the need to conduct laboratory research in the appropriate behavioral, evolutionary and ecological contexts [e.g. Roboreda et al., 1996; Gould and Gross, 2002]. A recent interesting study by LaDage et al. [2009] provides support for both claims. It shows that free-ranging mountain chickadees (Poecile gambeli) had more hippocampal new neurons than captive conspecifics. Further, it shows that depriving captive birds of the opportunity to engage in spatial memory use via food caching and retrieval resulted in significantly fewer new neurons in their hippocampus, compared with captive conspecifics that were allowed to engage in spatial memory use.

Stress is known to affect neurogenesis [e.g. Gould and Cameron, 1996; Gould et al., 1998]. Therefore, the finding that neuronal recruitment into the hippocampus is more pronounced under natural conditions can be related to stressful conditions that may exist in captive birds. However, evidence for the negative effect of stress on neurogenesis comes only from mammalian literature [see the review by Lucassen et

Highlights and Perspectives on Evolutionary Neuroscience

Brain Behav Evol 2010;75:86-87

DOI: $\underline{10.1159 / 000306483}$

\title{
Wild Neurogenesis
}

\author{
Anat Barnea \\ Department of Natural and Life Sciences, The Open University of Israel, Raanana, Israel
}

al., 2008], and therefore we do not know if this is also the case in birds. Moreover, basal corticosterone levels have been found to be very similar in adult free-ranging and aviary-held black-capped chickadees [unpubl. observations, see Barnea and Nottebohm, 1996]. Hence, stress might not be the only explanation for the lower numbers of new hippocampal neurons observed by LaDage et al. [2009] in the brains of captive birds.

An alternative possibility is that the significant factor which affected their hippocampal new neuronal recruitment was the fact that the 3-dimensional environment and spatial challenges that they encountered in the lab were much simpler than those experienced by their free-ranging conspecifics. Just a few obvious examples: the home range of a wild bird is incomparably larger than a testing room in the lab; the number of trees, potential caching sites, and actual caches that a bird uses and has to remember are also much more numerous. These spatial components in the bird's environment require adequate and updated spatial maps in its brain. In addition, in the natural world, spatial demands are not restricted to foodcaching behavior. Birds have to acquire and process much additional spatial information that relates, for example, to locations of roosting sites, alternative food sources, locations of predators, and more. Hence, it is simplistic to discuss differences in neuronal recruitment in the hippo- campus of free-ranging versus captive birds only in relation to differences in their ability to engage in food-storing behavior. Rather, we have to take into account the possibility that the higher neuronal recruitment in the free-ranging birds reflects the overall greater, more complicated, numerous and diverse memory-based challenges and demands with which they have to cope.

These thoughts raise a few questions. First, if we accept the importance and the need to conduct research under natural settings, can we control the conditions enough to get interpretable data? This is probably impossible. Despite this obstacle, studies on free-ranging species are still invaluable for understanding how neurogenesis and brain plasticity benefit animals in their struggle for survival. I agree with Armein et al. [2008], who advocate carrying out studies that deal with animals that live their lives in the context of what they were actually selected to do. Support for this view comes from LaDage et al. [2009], as well as from our previous work [Barnea and Nottebohm, 1994], which both demonstrate that data from laboratory studies provide an underestimate of the real magnitude of these phenomena. Hence, the scientific community should appreciate the need for conducting research under natural conditions and favor field studies by allocating enough funds to support and encourage such studies.

\section{KARGER \\ Fax +4161306 1234 E-Mail karger@karger.ch} www.karger.com www.karger.com/bbe
Anat Barnea

Department of Natural and Life Sciences

PO Box 808, 1 University Rd., The Open University of Israel

IL- 43107 Raanana (Israel)

Tel. +972 9778 1753, Fax +9729778 0661, E-Mail anatba@openu.ac.il 
Nevertheless, many aspects of neurogenesis and neuronal recruitment and survival can clearly only be investigated in a laboratory setup, under precise and controlled conditions. Hence, if we agree that both laboratory and field studies are essential, the choice depends on the questions asked and the aspects investigated. The elegant comparison in the LaDage et al. [2009] study between captive birds which were either allowed or denied food-caching spatial memory experiences, and the careful controls which were carried out, is a good example of the benefit that can be gained from laboratory experiments.

Therefore, the second question that should be raised is how closely can enriched environmental conditions in the lab resemble wild ones? Obviously, a laboratory setup, as enriched as possible, will not challenge the animal and its brain to the same extent that its natural habitat does. Also, it is probably easier to enrich the laboratory environment of small animals (e.g. rodents) than of bigger ones (e.g. primates). A partial solution, especially with small animals, is to use semi-natural conditions. Applying such an approach in the LaDage et al. [2009] study would have enabled them to avoid a few caveats and doubts. For example, since chickadees are social birds, it would have been better to house them in a group, rather than individually. Isolation may affect their behavior and potentially put them under stress, especially since they were kept isolated for a few months. Possible stress, in turn, might indirectly affect neurogenesis and/ or neuronal survival. Isolation might also affect new neuronal survival in the hippocampus, as we have previously shown in zebra finches [Barnea et al., 2006]

Another way to preserve more natural environmental factors is keeping the animals in outdoor aviaries. For example, while LaDage et al. [2009] maintained a natural light schedule, the birds probably did not experience the wide changes in temperatures between September and January that their free-ranging conspecifics did. We do not know whether ambient temperatures affect neurogenesis and/or neuronal survival, but one cannot rule out such an effect, even indirectly, via hormonal or metabolic changes in response to temperatures. Moreover, outdoor aviaries could provide animals with many other environmental stimuli, which might affect their well-being and biological processes.
Summing up, the LaDage et al. [2009] paper demonstrates the importance of studying neurogenesis and neuronal replacement under natural conditions and not only in the laboratory. Promoting studies on natural populations should be a major aim in the study of brain plasticity, and should be regarded as an important complement to laboratory experiments. Moreover, studying natural populations might increase the diversity of species and environmental conditions tested in association with neuronal replacement. This is important to provide consistent results that will help to determine the function of this phenomenon.

\section{Acknowledgments}

I would like to thank Georg Striedter for his good advice and patience, and Gila Haimovic for editing the text. Thanks are also due to the funding agencies that supported some of my studies which are cited here: The Institute of Psychobiology in Israel, The Israel Science Foundation (grant No. 481/04), and the Open University Research Fund.

\section{References}

Amrein I, Lipp HP, Boonstra R, Wojtowicz JM (2008): Adult hippocampal neurogenesis in natural populations of mammals; in Gage FH, Kempermann G, Song H (eds): Adult Neurogenesis. New York, Cold Spring Harbor Laboratory Press, pp 645-659.

Barnea A (2009): Interactions between environmental changes and brain plasticity in birds. Gen Comp Endocrinol 163:128-134.

- Barnea A, Mishal A, Nottebohm F (2006): Social and spatial changes induce multiple survival regimes for new neurons in two regions of the adult brain: an anatomical representation of time? Behav Brain Res 16:63-74.

Barnea A, Nottebohm F (1996): Recruitment and replacement of hippocampal neurons in young and adult chickadees: an addition to the theory of hippocampal learning. Proc Natl Acad Sci USA 93:714-718.
Barnea A, Nottebohm F (1994): Seasonal recruitment of hippocampal neurons in adult free ranging black-capped chickadees. Proc Natl Acad Sci USA 91:11217-11221.

Boonstra R, Galea MS, Wojtowicz JM (2001): Adult neurogenesis in natural populations. Can J Physiol Pharmacol 79:297-302.

Gould E, Cameron HS (1996): Regulation of neuronal birth, migration and death in the rat dentate gyrus. Dev Neurosci 18:22-35.

- Gould E, Tanapat P, McEwen BS, Flugge G, Fuchs E (1998): Proliferation of granule cell precursors in the dentate gyrus of adult monkeys is diminished by stress. Proc Natl Acad Sci USA 95:3168-3171.

Gould E, Gross CG (2002): Neurogenesis in adult mammals: some progress and problems. J Neurosci 22:619-623.
LaDage LD, Roth TC 2nd, Fox RA, Pravosudov V (2009): Ecologically relevant spatial memory use modulates hippocampal neurogenesis. Proc R Soc B 277:1071-1079.

Lucassen PJ, Oomen CA, van Dam AM, Czeh B (2008): Regulation of hippocampal neurogenesis by systemic factors including stress, glucocorticoids, sleep, and inflammation; in Gage FH, Kempermann G, Song H (eds): Adult Neurogenesis. New York, Cold Spring Harbor Laboratory Press, pp 363-395.

Nottebohm F (2002): Why are some neurons replaced in adult brain? J Neurosci 22:624628.

Reboreda JC, Clayton NS, Kacelnik A (1996): Species and sex differences in hippocampus size in parasitic and non-parasitic cowbirds. NeuroReport 7:505-508. 